\title{
Lunar Observation Support System Using Smartphone AR for Astronomy Education
}

\author{
http://dx.doi.org/10.3991/ijim.v8i1.3457 \\ K. Tian ${ }^{1}$, M. Endo ${ }^{2}$, M. Urata ${ }^{1}$, K. Mouri ${ }^{3}$ and T. Yasuda ${ }^{1}$ \\ ${ }^{1}$ Nagoya University, Nagoya, Japan \\ ${ }^{2}$ Chukyo University, Toyota, Japan \\ ${ }^{3}$ Nagoya City Science Museum, Nagoya, Japan
}

\begin{abstract}
It is possible to improve learners' understanding of the concept of lunar phases by using Observation based Learning (OBL). In this research, Smartphone Augmented Reality (AR) technology was used to develop a multiviewpoint AR-based mobile learning (M-VARML) system for moon observation that can be used in the real world environment. An experiment was conducted to evaluate the usefulness of our system. Participants were given five moon observation tasks, which they had to perform manually and using the M-VARML system. After each task, they were given follow-up questionnaires on the usefulness of the functions in the system. Finally, they were asked about the operational usability and the effect our system had in motivating them to pursue lunar observation. The results show that the M-VARML system is effective in improving the observation and learning of students and in enhancing their motivation to pursue lunar learning.
\end{abstract}

Index Terms-Smartphone Augmented Reality, Lunar Observation, Lunar Phase, Astronomy Education.

\section{INTRODUCTION}

Lunar phases are astronomical phenomena that we observe in our daily life. Yet, it may be one of the most difficult concepts to teach in astronomical education [1]. In a survey conducted in Japan, over $50 \%$ of pupils had difficulty in understanding lunar phases [2]. In addition, it is reported that only $30 \%$ undergraduate students could explain the mechanism of lunar phases [3]. In order to help learners grasp this abstract concept, research has been conducted to study the development and usefulness of educational materials for lunar phase learning using several different approaches. One type is the virtual learning environment. For example, a learning system is available that allows the user to manipulate astronomical models of the sun, earth, and moon which exist as visible, tangible bodies [4]. The tangible learning system, titled "The Phase of the Moon," was implemented in a six-grade elementary school science class unit. A 3D animation moon phase concept system has been applied to science learning in the fourth grade [5]. Several issues, however, arise with a virtual learning environment. For example, much of the research is confined to restricted locations such as computer rooms or laboratory. Lack of outdoor learning seriously limits students from clearly understanding the position and movement of moon phases in the real sky. A virtual learning system is abstract and difficult to apply to the real world environment. For example, in one study indicated that after students obtained elevation angle of the moon information from a virtual learning system, it was difficult for them to measure the actual elevation angle of moon in the real world [6]. Thus, the positive effects of virtual learning environments are limited in motivating and learning about lunar phases.

Planetarium curators believe that the most important element in astronomy education is observing real stars outdoors [7]. It is important to observe real objects and real phenomena to learn science; this helps the learners experience and understand the target objects or phenomena in their real world with its surrounding conditions and sceneries [8]. Observation-based learning (OBL) is one such kind of learning approach that meets the requirements of experiential learning; it can be employed in the real world to circumvent issues that exist in the virtual learning environment. Suzuki confirmed that on-going observation and conversation could enhance college students' ability to understand the phases of the moon [9]. An activity that could greatly aid a student in lunar phase learning is observing the moon in sky. Through observations, a student can understand the rising and setting of the moon. Further, by daily observation of lunar phases, they can comprehend the periodic waxing and waning of the moon and understand the relationship among the sun, earth, and moon. An approach to OBL in lunar education includes using an application on mobile device. A lunar observation support system that can be used with mobile phones was developed [10]. This system enables students to observe the moon in open air and send observational data through their mobile phones to the server. However, this system had issues in the real world environment. Observation of the moon is often restricted by time, place, and weather. On cloudy or rainy days, the observer failed to see the moon. Furthermore, in cities, it is often inconvenient for observers to view the lunar phases due to high rises or light pollution. Learners could not find time on thirty days to completely observe a lunar cycle because of their busy schedules. One typical solution is to use Augmented Reality (AR) technology to address these issues. Augmented Reality (AR) is a variation of Virtual Reality (VR); it adds virtual information to the real world and interacts with it. Kikuo and Tomotsugu [11] consider AR as a new pattern that could be applied in teaching that has great potential for future development. This new and unique teaching method and strategy can help learners with little computing experience to interact easily. Interest in mobile AR applications has grown with the dramatic increase in the usage of smart phones in the past few years. Present day smart phones and tablets integrate fast processors with 
graphics hardware, large touch screens, and onboard sensors (GPS, compass, and accelerometers) to create gadgets that are ideal for indoor as well as outdoor $\mathrm{AR}$ experiences, such as, CityViewAR [12].

There are a number of popular AR astronomy applica tions, such as Google Sky Map, Star Walk, and Stellarium Mobile. These applications utilize the Smartphone's GPS and sensors to locate the stars. These applications name the star that is being viewed through the phone. However, the purpose of these applications are not designed for lunar learning and teaching and cannot meet the learning goal of lunar phases; for example, they have not dynamic latitude and longitude line to help students to study the azimuth and elevation angle of moon in the sky. In addition, there are no functions that show one-month moon orbit, shape, and age in the AR view. Most application AR views do not have a camera view, and thus a visual view in the real world environment cannot be obtained. Further, these applications do not provide a function that explains the mechanism of lunar phases, as it relates to everyday observation of lunar phases.

Setozakai [13] proposed that it is necessary to help learners to understand the astronomy topics from multiviewpoints, namely the geocentric model and the heliocentric model viewpoint. It states that it is important to switch between the two viewpoints to understand astronomy subjects. Based on Setozakai's theory, Kawasaki [14] indicated that it is important to observe the appearance of moon from the earth viewpoint, and, at the same time, to understand the relationship between the sun, earth, and moon from the viewpoint with sun as the center of the universe to study the mechanism of lunar phases. Therefore, it is optimal to construct a multi-viewpoint observation environment for students to observe lunar phase in the real world environment.

In this research, Smartphone AR technology was applied to develop a multi-viewpoint AR-based mobile learning system for supporting moon observation that can be used anytime and anywhere, irrespective of the weather conditions. In order to assess the learning effect, usability and motivational effect of this system, a task-based experiment was conducted. Participants were given five moon observation tasks to perform manually and using MVARML system. After each task, they were given followup questionnaire about the usefulness of the functions in the system. Finally, they were asked about the operational usability and the learning attitude of system. The results show that the M-VARML system enhances a learner's learning ability for lunar observation. It has high motivational effect on lunar observation and operational usability.

\section{RESEARCH METHOD}

This section explains the learning goal of the system, the system design, the system interface, and its functionality.

\section{A. Learning Goal of Lunar Observation}

The proposed system was developed as an educational tool for lunar phase observation. The learning functions of the system are based on learning indicators in the student's science course unit, titled "Lunar Phase." This course set the learning goals of moon observation of MVARML as follows:
(1) Observe the moon, its azimuth and elevation angle in the sky every day.

(2) Observe the moon every day to witness it rising in the east and setting in the west.

(3) Observe the moon every day to understand the periodic waxing and waning of moon in 30 days.

(4) Observe the azimuth and altitude, changing of the shape of the moon by observing the moon at the same time in 30 days.

(5) Understand the positional relationship among the sun, moon, and observer.

(6) Understand the positional relationship among the sun, earth, and moon from the viewpoint with sun as the center of the universe.

(7) Understand the relationship between moon age and shape of lunar phases.

\section{B. System Outline}

The M-VARML System allows learners to observe the appearance of moon from the earth viewpoint, at the same time, understand the relationship of sun, earth and moon from the sun-center of universe viewpoint to study the mechanism of lunar phase. The outline of system is as shown in the Fig.1. The learners could switch the two viewpoints to observe moon and achieve the learning goal of moon that we have stated.

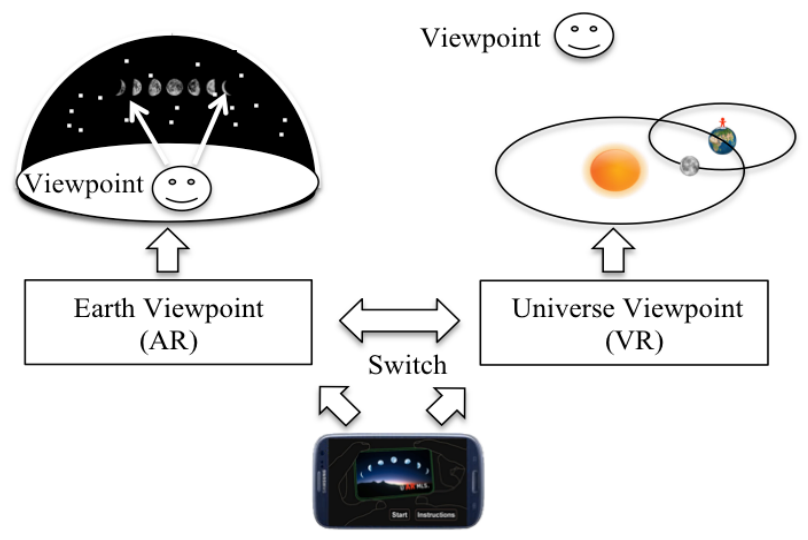

Figure 1. Outline of M-VARML

The M-VARML system has two main views: the AR view and the Universe View. In order to achieve the learning goals of lunar phase observation (see Section IIA), we set the following specifications of the learning environment in M-VARML system:

(a) The azimuth, elevation angle, shape, moon age of moon can be observed in the real sky through the AR view function.

(b) The azimuth, altitude of sun can be observed in the real sky through the AR view function.

(c) The orbit of moon between 1:00 AM and 12:00 PM can be observed in the real sky using the "One Day" function.

(d) The periodic waxing and waning of the moon in the sky can be observed through the "SetDay" function.

(e) The relationship between moon age and the shape of moon can be observed through the "SetDay" function.

(f) The positional relationship among the sun, observer, and moon can be understood through the AR view. 


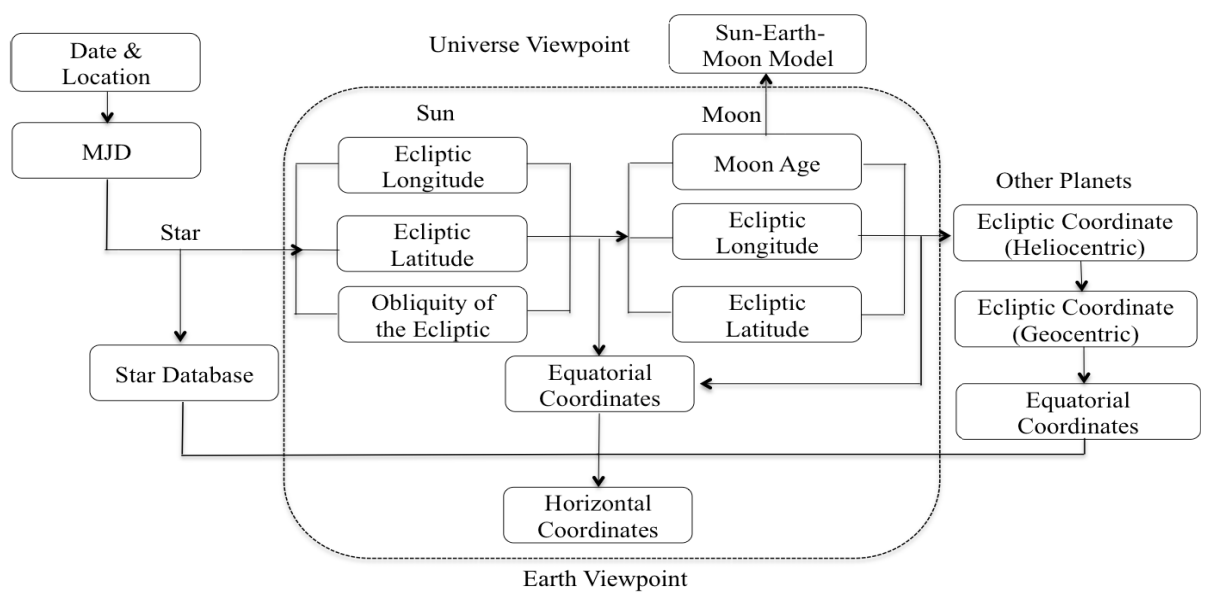

Figure 2. Astronomy simulation model

(g) The relationship among the sun, earth, and moon with respect to the universe can be understood through the universe view function.

In the above specifications, the specification (a) helps to achieve the learning goal (1). The specification(c) helps to achieve the learning goal (2). The specification (d) helps to achieve the learning goal (3) and (4). The specification (e) helps to achieve the learning goal (7). The specification (b) and (f) help to achieve the learning goal (5). The specification (g) helps to achieve the learning goal (6).

\section{Astronomy Simulation Model}

The M-VARML system is based on the astronomy model that the earth revolves around the sun, and moon revolves around the earth while rotating on its own axis. Our research group has been focusing on the simulation of astronomical phenomenon for a long time. Based on the astronomy simulation model, which is as shown in Fig.2, several astronomy education system [15] \& [16] have been developed. In this research, we employ the astronomy simulation model to develop the M-VARML system for supporting moon observation from multiviewpoints.

\section{AR View Function}

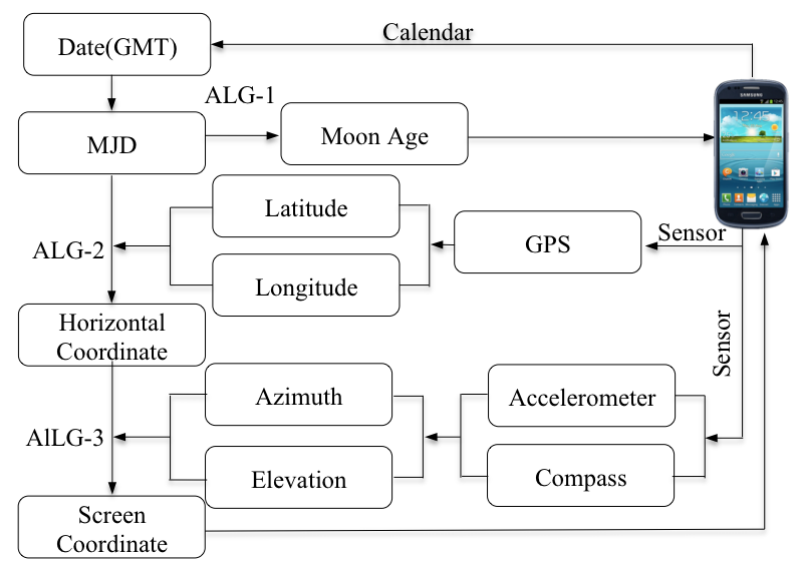

Figure 3. Procedure for calculating moon position in AR view

The M-VARML system shows virtual moon images overlaid on a live video camera background, making the virtual moon image appear in the real world. To visualize the moon correctly in the AR view, it is necessary to get real-time data from the Smartphone through built-in sensors. This is then combined with the astronomy simulation model (see Fig.2) to calculate the screen coordinates of the visual moon in AR view correctly registered to the real sky, which users observe in the sky.

The procedure for calculating the position of the moon is showed in Fig.3. Firstly, it is necessary to obtain realtime date and time from the calendar of the Smartphone and to calculate the Modified Julian Day (MJD) [17] based on the acquired date and time. Then the longitude and latitude data is obtained using GPS sensor, which is responsible for tracking the geographical position of the Smartphone. Combining these data with the horizontal coordinates algorithm (ALG-2), the moon age, azimuth, elevation of the real-time moon and sun can be computed. The azimuth and elevation data is the horizontal coordinate data of the moon in the sky. This is then used to derive the data of the screen coordinate of the moon and register the moon in the $\mathrm{AR}$ view, corresponding to the actual moon in the sky. The accelerometer sensor and compass sensor are used to measure the viewing direction and elevation angle of the Smartphone device that the user is using to observe the moon in the sky. Based on this tracking information, we use the screen projection algorithm (ALG-3) to convert the horizontal coordinates to a screen coordinates to locate the moon in the AR view. After this procedure, the virtual moon is shown in the AR view appropriately maps the moon from the real sky.

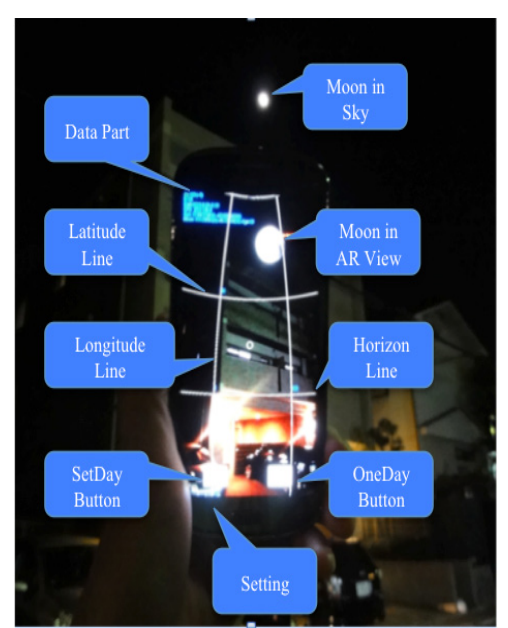

Figure 4. AR view interface 


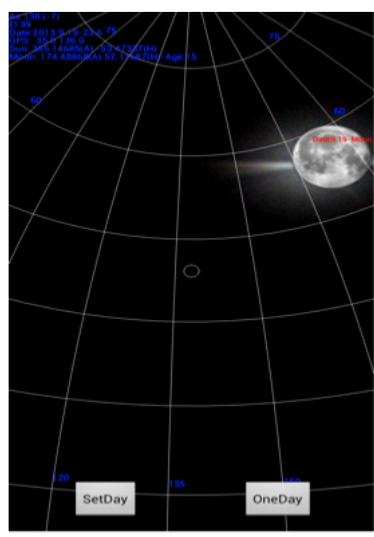

(a) Moon

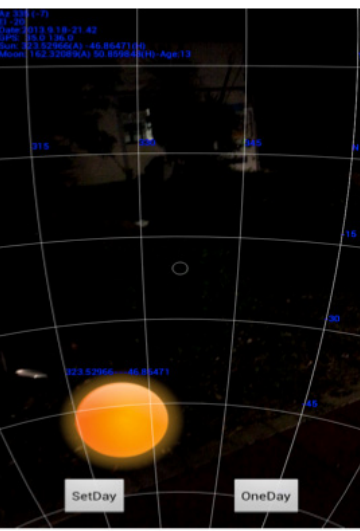

(b) Sun

Figure 5. Moon and Sun in AR view

The complete AR view interface of M-VARML is shown in Fig.4. The main visual components overlaid in the AR view include the data part, visual moon image, latitude line, longitude line, horizon line, and two buttons. The data part shows the data of orientation, elevation angle of mobile device, GPS, date, time, azimuth (sun and moon), elevation (sun and moon), and moon age. The horizontal line is the zero degree on the latitude line, indicating the horizon in the real world. N, E, S, W indicates the direction that the Smartphone device is pointing to in real world, corresponding to the north, east, south and west directions, which, on combining with latitude and longitude line helps learners search and observe the moon and sun as shown in Fig.5. In addition, learners could modify the setting including the degrees of latitude and longitude, size of the text, and color, etc., from the setting interface.

The "OneDay" function meets the specification of learning environment (c). After the users touch the "OneDay" button, as shown in Fig.6 (a), the one-day moon orbit will be displayed in the AR view as shown in Fig.6 (a). The learner rotates the mobile device to observe moon's orbit from 1:00 am to $12: 00 \mathrm{pm}$. It takes the learners a few minutes to observe the moon rising in the east and setting in the west, thereby assisting learners to achieve the learning goal (2).

The "SetDay" function in the AR view meets the specification-learning environment (d) \& (e), and the interface is as shown in Fig.7. Users touch the "SetDay" button to step into the "SetDay" interface as shown in Fig.7 (a). Users can choose the number of days of the moon from 1 to 30 days. For example, if a learner selects 30 days of the lunar phase, the lunar phases of the next 30 days will be overlaid in the AR view. In this view, the learners can observe the periodic waxing and waning of the moon, the orbit, azimuth, elevation angle, age, and shape of consecutive moons in the sky in a particular location at once. This merely takes a few minutes to complete the lunar cycle observation. This function helps learners to achieve the learning goal (3), (4), and (7).

Through the AR view function, learners not only could construct a visual feeling for lunar observation in the sky, but also could complete the learning goal of lunar observation from earth viewpoint at anywhere, anytime irrespective of the weather restriction in a short time.

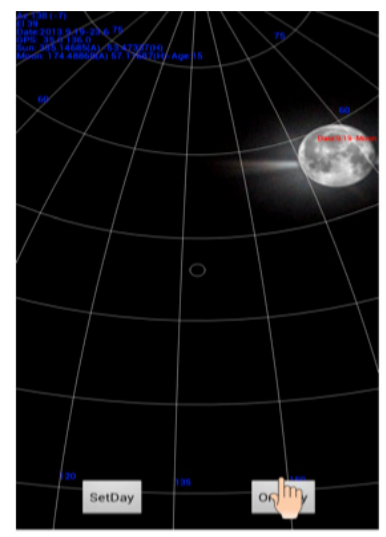

(a)

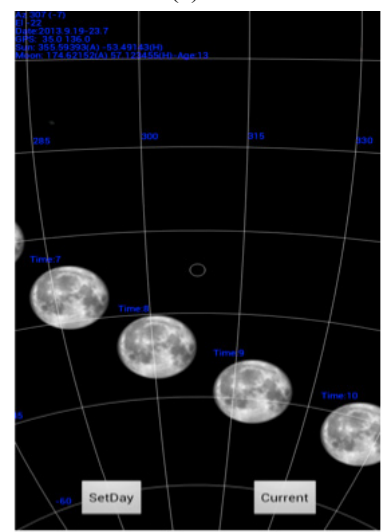

(c)

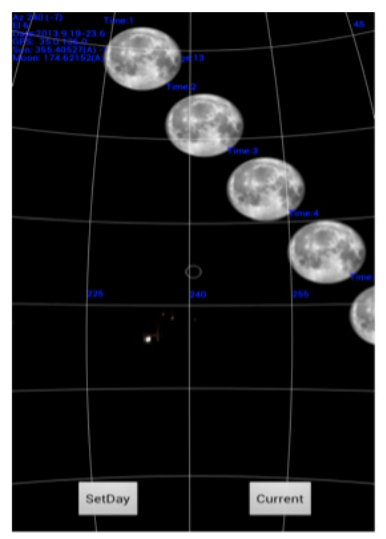

(b)

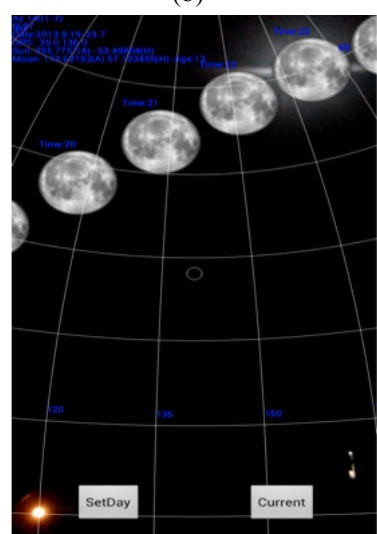

(d)
Figure 6. Observe one-day moon orbit in AR view

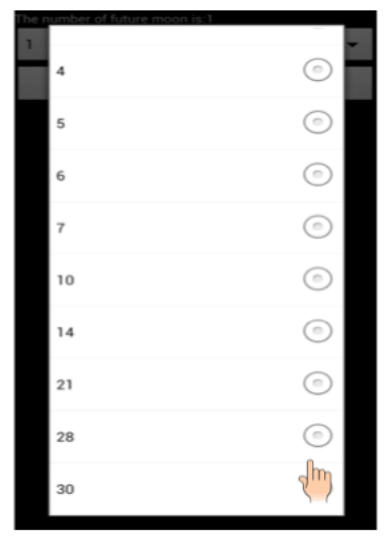

(a)

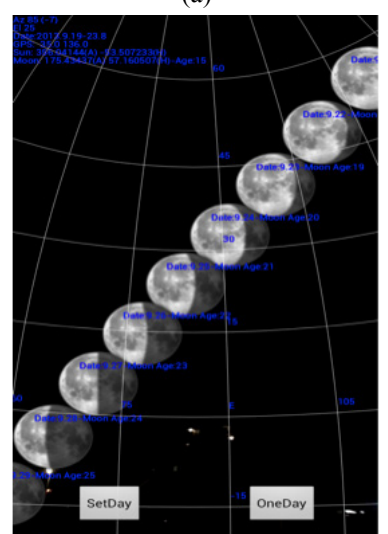

(c)

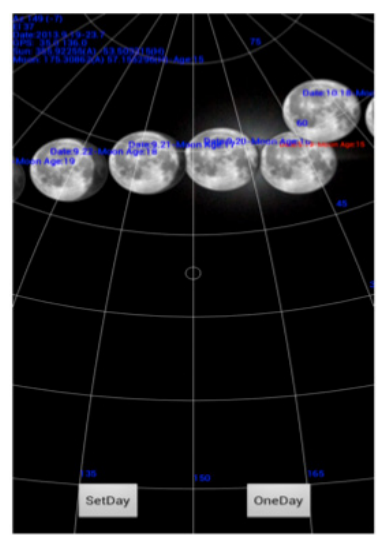

(b)

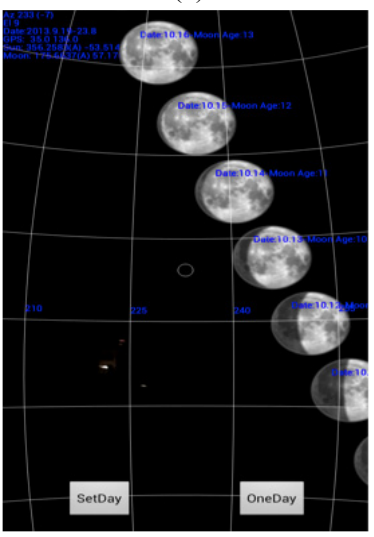

(d)
Figure 7. Observe waxing and waning of moon in AR view 


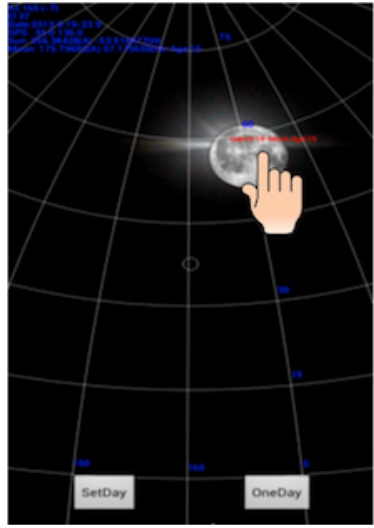

(a)

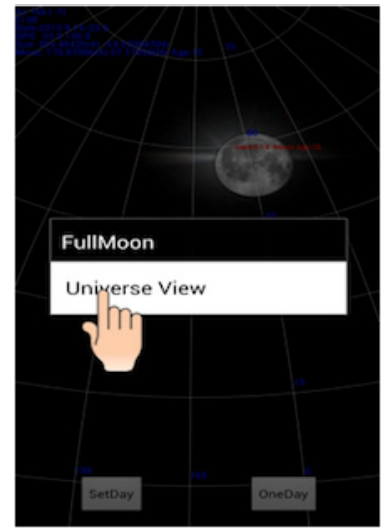

(b)

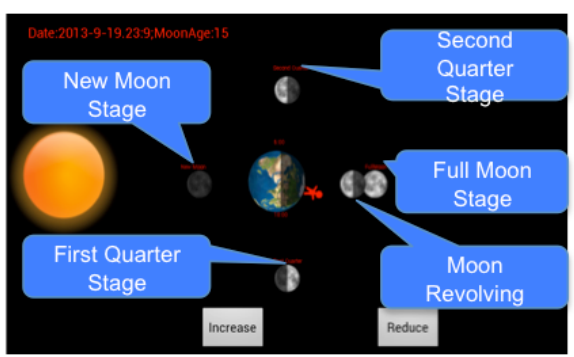

(c)

Figure 8. Step into the universe view
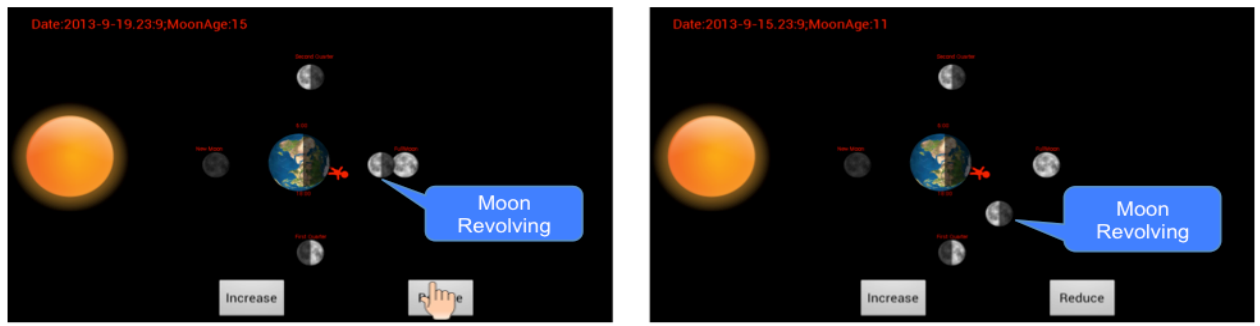

(a) Operating the increase button
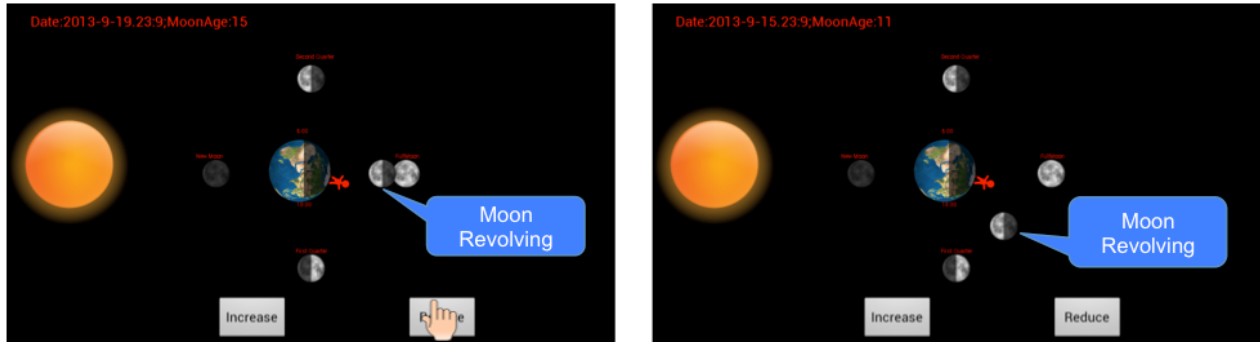

(b) Operating the reduce button

Figure 9. Interactive interface of universe view

\section{E. Universe View Function}

Learners observing the phases of the moon often wonder what causes this moon phase. The best way to understand the phases of the moon is to examine an earthmoon-sun view. We used the sun-earth-moon orbit astronomy model to develop the Universe view function. When the user touches the current moon image in the AR view, the user can see an item named Mechanism. Onselecting this item, the user steps into the Universe view interface as showed in Fig.8.

The lunar phases are due to how the sun illuminates the moon and the relative positioning of the earth, moon and sun. Fig. 8 shows that the moon revolves around the earth while the earth is rotating on it axis to give us day and night. The person icon on the earth indicates a user standing on the earth observing the moon. It takes the moon about 30 days to revolve around the earth. During those 30 days, as the moon orbits the earth, it grows in size from a new moon, to first quarter, to full moon. It then decreases in size in reverse order to last quarter and then backs to the new moon. Thus, in the Universe View, we visualize four key moon phases of the moon orbit. As shown in Fig.8(c), Thus, in the Universe View, we visualize four key moon phases of the moon orbit.
As shown in Fig.8(c), when users observe the actual moon in the sky, if they know that the moon age is 15 that particular day, and they see the proportion of the visible moon from the earth viewpoint, they can identify the relationship among the sun, earth, and moon through the Universe view function. Users discern that the moon is at the full moon stage.

In the AR view function, users can observe the moon at the same location and time in 30 days. We have also provided users with an interactive function to see the relationship among the sun, earth, and moon at the same location and time during 30 days in the Universe view. Learners can use the increase (see Fig.9 (a)) and reduce buttons (see Fig.9 (b)) to experience it. The Universe view meets the stated specification of the learning environment (g). This improves their knowledge regarding the mechanism of lunar phases.

\section{SYSTEM IMPLEMENTATION}

The M-VARML system is developed on the Google Android operating system and software development kit. The developing code toolset is Eclipse, ADT, and Android 2.1 SDK. The programming language is Java. The application is supported by a range of smart phones and tablet devices that run on Android 2.1 or higher, and have the required sensors (i.e., camera, GPS, electronic 
compass, and accelerometer). Our M-VARML was implemented on an Android Smart phone. The mobile device was a Samsung Galaxy S3.

\section{EXPERIMENT DESIGN}

For this research, two experiments were conducted to confirm the usefulness and usability of the system; the number of participants in each experiment is 10 (18 males, and 2 females, most of whom were in their twenties). They were mainly undergraduate and graduate students from the Nagoya University participated in the two experiments. It was verified that they had an interest in lunar phase observation. The first experiment [18] was learning goal experiment; the other one was task-based experiment in which participants were given specific tasks. In the first experiment, the learning goals of system (See Section II-a) were explained to the learners, and they were instructed on how to use the M-VARML system can be used to observe lunar phases in the real world environment. After the subjects used the system, they answered a questionnaire. The results of questionnaire showed that the system was helpful to learners in observing the lunar phase. However, interviews with participants indicated that they found the various functions of system perplexing and they were unable to grasp the usefulness of each function in the system.

Therefore in the second experiment of the present study, participants were given five tasks, which were based on the aforementioned learning goals of lunar phase observation (see Section II-a), the tasks are listed in Table I. and the purpose of these five tasks is to evaluate the usefulness of each function in system. Fig.11 shows the relationship between the tasks and the questions. Consider task 2 as an example; this task was to evaluate the usefulness of the "OneDay" function, which helps learners to achieve the learning goal (2).

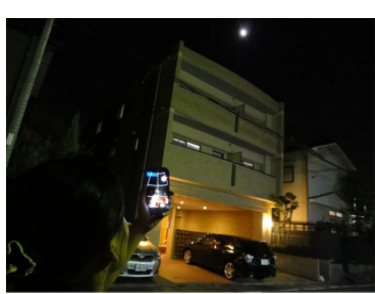

(a)

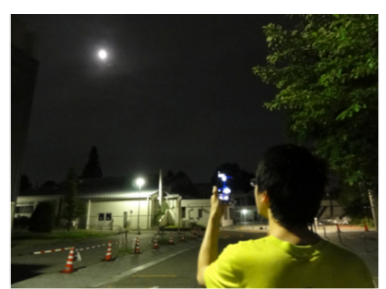

(b)
Figure 11. Experimental situations

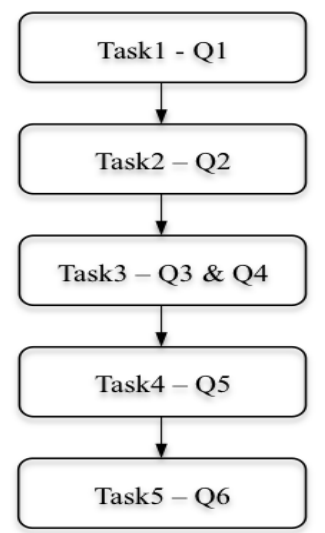

Figure 11. The relationship of the task and questions about usefulness.
TABLE I.

EXPERIMENTAL TASKS FOR LIST

\begin{tabular}{|c|c|}
\hline No. Items & Contents \\
\hline Task1 & $\begin{array}{l}\text { Firstly, participants use the M-VARML system to } \\
\text { search and observe real time moon to understand its } \\
\text { azimuth and elevation angle without the latitude } \\
\text { and longitude line in the AR view. Then users } \\
\text { choose the function that shows latitude and } \\
\text { longitude line in AR view. Lastly, the participants } \\
\text { use the system again to search the moon and } \\
\text { observe the azimuth and elevation angle of real } \\
\text { time moon in the sky. }\end{array}$ \\
\hline Task2 & $\begin{array}{l}\text { Initially, the participants guess the one-day moon } \\
\text { orbit and use their fingers to draw the line of one- } \\
\text { day moon orbit in the sky. In order to confirm their } \\
\text { drawings, they use the "OneDay" function to } \\
\text { observe the one-day moon orbit in the AR view. }\end{array}$ \\
\hline Task3 & $\begin{array}{l}\text { Firstly, the users guess the one-week moon orbit, } \\
\text { the shape and age of the moon. Then, they use their } \\
\text { fingers to draw the line of one-week moon orbit in } \\
\text { the sky and draw the shape of after one-week moon } \\
\text { on paper with a pen. In order to confirm their } \\
\text { drawing, they use the "SetDay" function and } \\
\text { choose seven days moon phase to observe the } \\
\text { future one- week moon orbit, shape, and age in the } \\
\text { AR view. Then the users guess thirty days of the } \\
\text { moons orbit and its shape, and then they use their } \\
\text { fingers to draw the line of } 30 \text { days moon orbit. } \\
\text { Finally, they choose } 30 \text { days lunar phase to observe } \\
\text { the } 30 \text { days moon orbit, shape, and age in the AR } \\
\text { view. }\end{array}$ \\
\hline Task4 & $\begin{array}{l}\text { Firstly, the participants guess the position of sun in } \\
\text { the sky by observing the shape of the moon. They } \\
\text { manually show the positional relationship among } \\
\text { the sun, observer, and moon. In order to confirm } \\
\text { the positional relationship, users use the system to } \\
\text { search the sun and moon in the AR view. }\end{array}$ \\
\hline Task5 & $\begin{array}{l}\text { Initially, the participants guess the relationship } \\
\text { among the sun, earth, and moon in the universe } \\
\text { view and draw the relationship of these objects on } \\
\text { paper with a pen, in accordance with the shape of } \\
\text { the moon. Then, they click the moon image to step } \\
\text { into the Universe view to verify the relationship } \\
\text { among the sun, earth, and moon. Then, they can } \\
\text { click on the increase and reduce buttons to explore } \\
\text { the relationship between the sun-earth-moon in one } \\
\text { month. }\end{array}$ \\
\hline
\end{tabular}

TABLE II.

QUESTIONNAIRE ABOUTY USEFULNESS

\begin{tabular}{|c|c|}
\hline No. Items & Contents \\
\hline Q1 & $\begin{array}{l}\text { The function of latitude and longitude line in } \\
\text { system is more helpful to me in understanding the } \\
\text { azimuth and elevation angle of real time moon in } \\
\text { the starry sky. }\end{array}$ \\
\hline Q2 & $\begin{array}{l}\text { The function of "OneDay" in system is helpful to } \\
\text { me in observing the movement orbit of one-day } \\
\text { moon in the starry sky. }\end{array}$ \\
\hline Q3 & $\begin{array}{l}\text { The function of "SetDay" in system is helpful to } \\
\text { me in observing the periodic waxing and waning } \\
\text { of moon in } 30 \text { days. }\end{array}$ \\
\hline Q4 & $\begin{array}{l}\text { The function of "SetDay" in system is helpful to } \\
\text { me in understanding the relationship between } \\
\text { moon age and shape of moon. }\end{array}$ \\
\hline Q5 & $\begin{array}{l}\text { The function of } \mathrm{AR} \text { view in system is helpful to } \\
\text { me in understanding the positional relationship } \\
\text { among sun-observer-moon in the real-world } \\
\text { environment. }\end{array}$ \\
\hline Q6 & $\begin{array}{l}\text { The function of Universe view in system is helpful } \\
\text { to me in understanding the positional relationship } \\
\text { among sun, earth, and moon from the universe } \\
\text { viewpoint. }\end{array}$ \\
\hline
\end{tabular}


TABLE III.

QUESTIONNAIRE ABOUT USABILITY AND SATISFACTION

\begin{tabular}{|c|l|}
\hline No. Items & \multicolumn{1}{c|}{ Contents } \\
\hline Q7 & It is easy for me to operate the system. \\
\hline Q8 & $\begin{array}{l}\text { It is interesting for me to use the system to observe } \\
\text { lunar phase. }\end{array}$ \\
\hline Q9 & $\begin{array}{l}\text { The system is helpful to me in enhancing my } \\
\text { motivation on moon observation. }\end{array}$ \\
\hline
\end{tabular}

These participants were asked to perform moon observation tasks in the real world environment. Some examples that were selected are shown in Fig.11. The tasks are listed in Table I. After each task was completed, the participant was given follow-up questions seeking the usefulness of the M-VARML system corresponding to the task. These questions can be found in Table II.

After completing all the five tasks and the corresponding questions about the usefulness, participants were give three questions and a free-response item on usability and satisfaction of the system. These questions can be found in Table III. All surveys used a five-point Likert-scale, the responses to which were coded as $1=$ strongly disagree through $5=$ strongly agree.

\section{EXPERIMENT RESULT}

\section{A. Usfulness of System}

The results of the survey pertaining to the usefulness of system during the task stage is as shown in Table IV. The mean of the questionnaire item Q1 was 4.60, showing that the users deemed the latitude and longitude line in AR view was helpful to them in observing the azimuth and elevation angle of the moon and sun in sky. During task 1, users agreed that the latitude and longitude line is helpful to them in constructing a visual feeling on azimuth and elevation angle in real sky. Therefore, for the usefulness of "OneDay" moon function (Q2), the participants confirmed that this function is very useful in observing the moon rise and set in a day since only one participant was able to draw the correct orbit line of one-day moon in the sky while performing task 2. Similarly, only one user could complete task 3 . Some participants even lacked any remote perception of the relationship between the moon age and its shape in the sky. Therefore, the participants conclusively agreed that the function of "SetDay" was helpful to them in observing the periodic waxing and waning of moon, and in understanding the relationship between the moon age and the shape of the moon. The mean of for Q3 and Q4 was 4.60 and 4.40 , proving the same. After completing task 4, most of the participants thought that the AR view was helpful in understanding the relationship between the sun, observer, and moon from the earth viewpoint, as is evident from the mean of 4.20 suggests for Q5. As all the questionnaire items (Q1, Q2, Q3, Q4, Q5) regarding the usefulness of the AR view, have a mean above 4.2 , it indicates that the AR view is very helpful to the learners in observing the lunar phase and is able to provide a visual learning experience in the real world. In addition, four participants succeeded in completing task 5; users thought that the Universe view function was helpful in understanding the relationship among the sun, earth, and moon from the universe viewpoint. However, as compared to the aforementioned AR view function, the mean of Q6 was just 3.60 since
TABLE IV.

RESULT OF THE QUESTIONNAIRE ABOUT USEFULNESS

\begin{tabular}{|c|c|c|c|c|c|c|}
\hline No. Items & $\mathbf{1}$ & $\mathbf{2}$ & $\mathbf{3}$ & $\mathbf{4}$ & $\mathbf{5}$ & Mean \\
\hline Q1 & 0 & 0 & 0 & 4 & 6 & 4.60 \\
\hline Q2 & 0 & 0 & 1 & 2 & 7 & 4.60 \\
\hline Q3 & 0 & 0 & 1 & 2 & 7 & 4.60 \\
\hline Q4 & 0 & 0 & 1 & 4 & 5 & 4.40 \\
\hline Q5 & 0 & 1 & 0 & 5 & 4 & 4.20 \\
\hline Q6 & 1 & 2 & 1 & 2 & 4 & 3.60 \\
\hline
\end{tabular}

TABLE V.

RESULT OF THE QUESTIONNAIRE ABOUT USABILITY AND SATISFACTION

\begin{tabular}{|c|c|c|c|c|c|c|}
\hline No. Items & 1 & 2 & 3 & 4 & 5 & Mean \\
\hline Q7 & 0 & 1 & 3 & 3 & 3 & 3.80 \\
\hline Q8 & 0 & 0 & 2 & 5 & 3 & 4.10 \\
\hline Q9 & 0 & 0 & 3 & 3 & 4 & 4.10 \\
\hline
\end{tabular}

most of participants considered that it was better to use the 3D space to show the relationship of sun, earth, and moon. Some of participants suggested that it would be better for new users if additional explanatory text were added in the Universe view to aid them in understanding the mechanism of lunar phases. From this analysis and the experimental results, we can draw the conclusion that the functions of our system are very useful for learners for lunar observation.

\section{B. Usability of System}

In term of interface designing, most of the users agreed that the system was easy to operate, with the mean of Q7 being 3.80, which is shown in Table V. They were able to operate the system to complete the moon observation tasks. The participants did report that the data in the AR view was a little hard to understand due to the usage of professional terms in that view. According to the interviews, it was reported that the size of the text should be larger or that a different set of colors would make the interface easier to understand.

\section{Satifsaction of System}

In addition, the participants appeared to be highly interested in using the system to observe lunar phase, with the mean of Q8 being 4.10. Participants agreed that the system is helpful to them in enhancing their motivation on moon observation in their daily life, which the mean of Q9 is 4.10 .

In the questionnaire we also asked question that required participants to freely write down their opinions. 2 participants mentioned it would be better if we highlighted the horizon line and 1 user suggested that we use the arrow guidance to find the moon. We also asked participants what new feature they would like to see in the system. 3 participants suggested that it would be better to have a full moon reminder function. Three 3 users asked if it would be possible to include tidal information related to the lunar phase.

\section{CONCLUSION}

In this study, we utilized the Smartphone AR technology and 2D content to construct multi-viewpoint observation environment for learners to observe lunar phases in the real-world environment without restrictions of weather, time, or place. The system has an AR view (to study the orbit, azimuth, elevation angle and circle of 
moon phases) and a Universe view (to help understand the mechanism of moon phases from the universe viewpoint) to support the learners with moon observation.

The results of the experiment revealed that each function in system was very usable and useful, these functions assisted learners in achieving the defined lunar observation learning goals using the M-VARML system. Therefore the system is helpful to learners to perform moon observation in the real world environment. Furthermore, the M-VARML system enhanced the learners' interest and motivated the learners to observe moon phases in their daily lives.

For future work, we plan to improve the interface design of the system so that even the learners with limited knowledge can use it and add more features, such as providing astronomy news about lunar phase. Further, our research group works in collaboration with Nagoya City Science Museum. Astronomy Curator plays a very important role in astronomy education. An important part of the future work includes discovery of ways to make best use of the knowledge of the curator in learning about the lunar phases. Our future challenges include the implementation of the M-VARML in Nagoya City Science Museum to test the learning effects of system.

\section{ACKNOWLEDGMENT}

The M-VARML system for lunar observation that was developed as result of this research is an important part of a project named "Astronomy Education Platform for Use in Smartphone Augmented Reality Technology" which cooperated with the Nagoya City Science Museum and Chukyo University in Japan. This project was supported by the Grants-in-Aid for Scientific Research Japan (No. 25280131). In addition, thanks to Mr. Tom Kabara of Mei Writing in Nagoya University for patiently guiding us in writing our paper.

\section{REFERENCES}

[1] R. Zheng and J. Olsen, "Developing the Lunar Phases Concept Inventory," Proceedings of the 2002 Physics Education Research Conference, 2002.

[2] H. Agata, "Science Education Collapse - A Survey about the Astronomy Education in Elementary School," The Astronomical Herald, vol.97, no. 12, pp.726-736, 2004.

[3] A. Ito, M. Chida, H. Tabara, "Variation of Astronomical Knowledge of University Students Revealed from Investigations Carried out in 1976 and 2006," Bulletin of Integrated Research Center for Educational Practice Faculty of Education, Utsunomiya University, vol.30, no.7, pp. 473-482, 2007.

[4] Y. Morita and N. Setozaki, "Practical Evaluation of Tangible Learning System: Lunar Phase Class Case Study," Proceedings of Society for Information Technology and Teacher Education International Conference, pp. 3718-3722, Mar, 2012.

[5] C. Ma, "Teaching Effects of 3D Animation Applied to Science Learning in the Fourth Graders-The Case of Moon-Phase Conception," M.S. thesis, National Taitung University, Taiwan, 2008.

[6] Y. Lin, "A study of AR-based Moon Observation System for Learning," M.S thesis, National Hsinchu University of Education, Taiwan, 2012.

[7] M. Kondo, M. Mouri, and Y. Yausda, "A Multiplatform Content Management System for Curators to Provide Teaching Materials in Astronomy Education," Journal of Theoretical and Applied Information Technology, vol. 4, no. 9, pp. 874-881, 2005.
[8] M. Soga, M. Ohama, Y. Ehara and M. Miwa, "Real-World Oriented Mobile Constellation Learning Environment Using Gaze Pointing," IEICE Transactions on Information and Systems, vol.94, no.4, pp. 763-771, Apr, 2011 http://dx.doi.org/10.1587/ transinf.E94.D.763

[9] M. Suzuki, "Conversations about the Moon with Prospective Teachers in Japan," Science Education, vol.87, no.6, pp. 1-13, 2003. http://dx.doi.org/10.1002/sce.10082

[10] H. Miyata, M. Suziki, M. Fukahori, and T. Akamastu, "Development and Educational Practice of a Lunar Observation Support System by Using Mobile Phones for Science Education," International Journal on Advances in Intelligent System, vol. 1, no. 1, pp. 1-10, 2008.

[11] M. Kikuo and K. Tomotsugu, "Augmented Instructions a Fusion of Augmented Reality and Printed Learning Materials," Proceedings of the fifth IEEE international Conference on Advanced Learning Technologies, pp. 213-215, July, 2005.

[12] G. Lee, A. Andreas, and A. Kim, "CityViewAR: A Mobile Outdoor AR Application for City Visualization," 11th IEEEE International Symposium on Mixed and Augmented Reality and Communication Engineers Technical Report, pp. 51-56, Dec, 2007.

[13] N. Setozakai, Y. MORITA, and T. TAKETA, "Study on Effective Use Scene of Multi Aspect Type VR Teaching Materials in Astronomical Education," The Institute of Electronics Information and Communication Engineers Technical Report, vol.107, no.391, pp. 51-56, 2007.

[14] T. Kawasaki, N. Iwane, and Y. Matsubara, "Development of a VR-based Astronomy Learning Environment using Intuitive Manipulation and Switching of the View corresponding to User's Body Movement," Japan Society for Educational Technology, vol. 34, no. 3, pp. 153-160, 2010.

[15] E. Akao, M. Endo, T. Naka, K, Iwazaki, K. Mouri and T. Yasuda, "Design and Development of Tablet-based Digital Teaching Material for Annular Eclipse Lectures based on Cooperation with a Museum," 2012 The Society of Socio-Informatics, pp.301-304, Sep, 2012.

[16] M. Ura, M. Endo, M. Yamada, S. Miyazaki, K. Iwazaki, K. Mouri and T. Yasuda, "A Smartphone Application for Searching Constellations Interactivity," Workshop on Advanced Image Technology Technical Program, pp.283-288, Jan, 2013.

[17] M. Jean, “Astronomical Algorithm," pp. 59-60, 1998, WillmannBell.

[18] K. Tian, M. Endo, M. Urata, K. Mouri and T. Yasuda, "Ubiquitous Augmented Reality Mobile Learning System for Supporting Moon Observation," International Conference on Information and Social Science, pp. 223-242, Sep, 2013.

\section{AUTHORS}

K. Tian is $\mathrm{PhD}$ Candidate in the Graduate School of Information Science at Nagoya University, Japan. He can be reached by tianke0711@gamil.com.

M. Endo is an associate professor in the School of Engineer at Chukyo University, Japan. He can be reached by endo@om.sist.chukyo-u.ac.jp.

M. Urata is an assistant professor in the Graduate School of International at Nagoya University, Japan. She can be reached by mayu@nagoya-u.jp.

K. Mouri is a curator of the Planetarium in Nagoya City Science Museum, Japan. He can be reached by mouri@nagoya-p.jp.

T. Yasuda is a professor in the Graduate School of Information Science at Nagoya University, Japan. He can be reached by yasuda@is.nagoya-u.ac.jp.

Submitted, November 13, 2013. Published as re-submitted by the authors 05 January 2014. 